\title{
A lopsided chemically distinct nucleus in NGC 5055^
}

\author{
V. L. Afanasiev ${ }^{1}$ and O. K. Sil'chenko ${ }^{2,3}$ \\ 1 Special Astrophysical Observatory, Nizhnij Arkhyz, 369167 Russia \\ 2 Sternberg Astronomical Institute, University Av. 13, Moscow 119992, Russia \\ ${ }^{3}$ Isaac Newton Institute of Chile, Moscow Branch
}

Received 23 November 2001/ Accepted 12 April 2002

\begin{abstract}
Kinematics and stellar population properties in the center of nearby Sbc galaxy NGC 5055 are studied with the Multi-Pupil Spectrograph of the $6 \mathrm{~m}$ telescope of the Special Astrophysical Observatory of Russian Academy of Sciences (SAO RAS). We confirm the rotation and stellar velocity dispersion asymmetries along the major axis reported earlier by other authors. We have found a resolved chemically distinct core in NGC 5055, with the magnesium-enhanced region shifted by $2 . \prime 5(100 \mathrm{pc})$ to the south-west from a photometric center, toward a kinematically identified circumnuclear stellar disk. Mean ages of stellar populations in the true nucleus, defined as the photometric center, and in the magnesium-enhanced substructure are coincident and equal to 3-4 Gyr being younger by several Gyr with respect to the bulge stellar population. A possible origin of the asymmetries in the center of NGC 5055 is discussed.
\end{abstract}

Key words. galaxies: individual: NGC 5055 - galaxies: nuclei - galaxies: stellar content galaxies: kinematics and dynamics - galaxies: evolution

\section{Introduction}

Very nearby luminous galaxies seem to be studied in detail because each of them has a long list of papers devoted to various aspects of its appearance. But a greater amount of information always reveals an extreme complexity and individuality of the target, so no galaxy is understood in detail. We will use NGC 5055 to illustrate this idea. The main characteristics of this galaxy are given in Table 1 .

A large nearby Sbc galaxy, NGC 5055 attracted the attention of investigators from the beginning of the quantitative extragalactic researches. Fish (1961) did photographic surface photometry of the galaxy, and Burbidge et al. (1960) obtained a rotation curve of the ionized gas with the slit aligned with the major axis of the galactic isophotes. Later, Bosma (1981) studied the rotation and structure of its neutral hydrogen disk and found that the HI disk was twice as extended as the stellar one and showed a noticeable warp beyond the boundaries of the optical image. Afterward kinematical and photometric

Send offprint requests to: O. K. Sil'chenko,

e-mail: olga@sai.msu.su

* Based on observations collected with the $6 \mathrm{~m}$ telescope of the Special Astrophysical Observatory (SAO) of the Russian Academy of Sciences (RAS) which is operated under the financial support of Science Department of Russia (registration number 01-43) and on data from the HST Archive.
Table 1. Global parameters of NGC 5055.

\begin{tabular}{lc}
\hline \hline NGC 5055 & \\
\hline Type $\left(\mathrm{NED}^{1}\right)$ & $\mathrm{SA}(\mathrm{rs}) \mathrm{bc}$ \\
$R_{25}, \mathrm{kpc}\left(\mathrm{LEDA}^{2}\right)$ & 17.2 \\
$B_{\mathrm{T}}^{0}\left(\mathrm{RC} 3^{3}\right)$ & 9.03 \\
$M_{B}(\mathrm{LEDA})$ & -21.27 \\
$B-V(\mathrm{RC} 3)$ & 0.72 \\
$V_{\mathrm{r}}(\mathrm{radio}, \mathrm{LEDA}), \mathrm{km} \mathrm{s}^{-1}$ & 503 \\
Distance (LEDA, $\left.H_{0}=75 \mathrm{~km} \mathrm{~s}^{-1} \mathrm{Mpc}^{-1}\right)$ & $9.2 \mathrm{Mpc}$ \\
Inclination (LEDA) & $55.5^{\circ}$ \\
$\mathrm{PA}_{\mathrm{phot}}(\mathrm{LEDA}, \mathrm{NED})$ & $105^{\circ}$ \\
$v_{\mathrm{m}}, \mathrm{km} \mathrm{s}^{-1}$ (LEDA) & 211 \\
$\sigma_{*}, \mathrm{~km} \mathrm{~s}^{-1}$ (Heraudeau \& Simien 1998) & $117 \pm 7$ \\
\hline${ }^{1}$ NASA/IPAC Extragalactic Database. \\
${ }^{2}$ Lyon-Meudon Extragalactic Database. \\
${ }^{3}$ Third Reference Catalogue of Bright Galaxies.
\end{tabular}

studies of NGC 5055 were repeated more than once, and often the results were controversial.

Firstly, the spiral arm classification of NGC 5055 remains ambiguous. In the optical Elmegreen (1981) and Elmegreen \& Elmegreen (1987) classified NGC 5055 as a flocculent spiral galaxy. But in the NIR $\left(K^{\prime}\right)$ - and COimages Thornley (1996) and Thornley \& Mundy (1997) 
saw two disconnected grand-design spiral patterns, inner and outer, with a boundary at $1.5-2.0 \mathrm{kpc}$ from the center.

Secondly, though the classification of NGC 5055 as Sbc is certain, we do not know yet if the galaxy is disk- or bulge-dominated. Thornley (1996), by decomposing the $K^{\prime}$-brightness profile into an exponential disk and a de Vaucouleurs' bulge, found that the bulge is almost everywhere more luminous than the disk, including the regions with grand-design stellar spiral arms. Fillmore et al. (1986) found that the disk of NGC 5055 dominates the bulge at $R \geq 7^{\prime \prime}$, but according to decomposition results by Baggett et al. (1998), the disk starts to dominate at $R=35^{\prime \prime}$. Determinations of the disk exponential scalelength range from 40" (Acaretta et al. 1996) to 98" (Kent 1987). Our experience of brightness profile decompositions with NGC 7331 (Sil'chenko 1999b) and NGC 7217 (Sil'chenko \& Afanasiev 2000) shows that when such a variety of decomposition results exists, a global disk may be readily divided into several decoupled segments. Two disconnected parts of the spiral pattern in NGC 5055 make this feasible.

The results of kinematical studies are also unusual. Fillmore et al. (1986) obtained long-slit cross-sections along the major and minor axes and compared the lineof-sight velocity distributions for the stars and ionized gas with the model predictions based on the brightness distribution. Both observed velocity profiles, and especially the one for the stars, appeared to be strongly asymmetric. The relative (rotation?) velocities of the stars to the east of the nucleus are twice as low as those to the west, and the corresponding stellar velocity dispersions differ in the opposite sense. Fillmore et al. (1986) note that the isophote shape in the center of NGC 5055 implies strong dust influence to the west and south-west of the nucleus; perhaps, on one (the eastern) side they saw the bulge and on the other (western) side the (warped) disk. However, simultaneously they detected "weaker absorption lines" just in the region where the kinematics implied the bulge; this combination seemed to them improbable. So the puzzle remains to be solved. Pismis et al. (1995) analysed ionized-gas line-ofsight velocity distributions along four different directions obtained under good seeing conditions $\left(1^{\prime \prime}-1^{\prime \prime} \cdot 3\right)$, and they also found a noticeable asymmetry along the major axis: within $R \approx 3^{\prime \prime}$ to the west of the nucleus there is an excess of relative gas velocity. The authors treat this excess as a manifestation of gas radial outflow due to mild nuclear activity - a rather strange interpretation considering that on the major axis the projection of radial velocities onto the line of sight is zero.

Among the other interesting features of NGC 5055 we must mention its ultraviolet nucleus. The galaxy is classified as a "UV-bright LINER" - within the heterogeneous class of LINERs, the "UV-bright" ones are usually thought to possess a non-thermal ionizing source. But in NGC 5055 the ultraviolet nucleus mapped by HST/FOC with high spatial resolution has appeared to be resolved: its diameter is $6 \mathrm{~h}^{-1} \mathrm{pc}$ and its absolute magnitude is $M_{B \text {, nuc }}=-11.2$
Table 2. 2D spectroscopy of NGC 5055 .

\begin{tabular}{lcclc}
\hline \hline Date & Exposure & Seeing & Sp. range & PA(top) \\
\hline $14 / 15.06 .99$ & $40 \mathrm{~min}$ & $1^{\prime \prime} .5$ & $4250-5600 \AA$ & $299^{\circ} .3$ \\
$15 / 16.06 .99$ & $45 \mathrm{~min}$ & $1^{\prime \prime} .2$ & $5850-7200 \AA$ & 293.7 \\
\hline
\end{tabular}

(Maoz et al. 1995), which implies that the UV nucleus of NGC 5055 may be a young stellar cluster.

Some years ago we (Sil'chenko 1994) compiled a list of galaxies that were candidates for possessing chemically distinct nuclei based on redder colours of the nuclei with respect to the bulges. NGC 5055 was not included in this list. But later we saw from the photometric survey of Heraudeau \& Simien (1996) that it has a very red colour, $V-I>1.5$, of the central region within $R=10^{\prime \prime}$. To check if this is a dust concentration or a chemically distinct nucleus, we needed panoramic spectral observations. We have observed NGC 5055 with the Multi-Pupil Fiber Spectrograph (MPFS) of the $6 \mathrm{~m}$ telescope in the framework of our program of searching for chemically distinct nuclei in spiral galaxies, and we found such a nucleus in this galaxy.

\section{Observations and data reduction}

In 1999 we undertook two-dimensional spectroscopy of NGC 5055 with the Multi-Pupil Fiber Spectrograph (MPFS) of the $6 \mathrm{~m}$ telescope of the Special Astrophysical Observatory (Nizhnij Arkhyz, Russia). Exposures were obtained in two spectral ranges: 4250-5600 A (blue-green), and 5850-7200 $\AA$ (red). Detailed information for the observations is given in Table 2. A grating of 1200 grooves per mm was used that provided a reciprocal dispersion of $1.35 \AA$ per pixel and a spectral resolution of $4 \AA$. A value for the seeing $F W H M=1^{\prime \prime} 2-1$.' 5 was estimated from an exposure of a double star, STF 1947, which was used to calibrate the orientation of our pupil frame on the sky.

These spectral observations were made with the new version of the panoramic spectrophotometer which became operational at the prime focus of the $6 \mathrm{~m}$ telescope at the end of 1997. With respect to the previous variants of MPFS (Afanasiev et al. 1990, 1996), the field of view is now increased and the common spectral range is larger due to the use of fibers: they transmit light from $16 \times 15$ square elements of the galaxy image to the slit of the spectrograph (240 fibers) together with the sky background taken 4.5 away from the galaxy itself (16 fibers). The size of one spatial element is $1^{\prime \prime} \times 1^{\prime \prime}$. At the exit of the spectrograph a $1024 \times 1024$ CCD registers all 256 spectra simultaneously. The primary reduction of the data is made within IDL. After bias subtracting, flatfielding, and one-dimensional spectra extraction from the CCD frame, we linearize and analyse each spectrum individually. The one-element spectral characteristics, such as flux in the continuum or in emission lines, redshift, and absorptionline indices are then combined into two-dimensional arrays corresponding to the galactic region under consideration using software developed earlier in the Special 
Astrophysical Observatory (Vlasyuk 1993) and our own programs. To calculate absorption-line indices and their errors we also used the program of Dr. Vazdekis. We obtained two-dimensional surface brightness distributions, velocity fields, and maps of stellar population characteristics. In the blue-green spectral range, we measured the absorption-line indices $\mathrm{H} \beta, \mathrm{Mgb}, \mathrm{Fe} 5270$, and Fe5335 in the Lick system (Worthey et al. 1994); to check the consistency of our measurements with the model indices calculated in this system (Worthey 1994), we regularly observed stars from their list (Worthey et al. 1994). The duration of exposure in the blue-green was chosen to be long enough to provide signal-to-noise ratios of about 100 (per Angstrom) in the nucleus and $\leq 20$ near the edges of the frames; the corresponding random error estimations made following Cardiel et al. (1998) range from $0.15 \AA$ in the center to $0.6-0.8 \AA$ for the individual spatial elements in the outer part. To keep a constant level of accuracy along the radius, we co-added the spectra in concentric rings centered on the nucleus, traced the radial variations of the azimuthally-averaged absorption-line indices, and compared them to the synthetic models of old stellar populations of Worthey (1994) and Tantalo et al. (1998). We estimate the mean accuracy of our azimuthally-averaged indices as $0.1 \AA$. Besides index mapping, we use our bluegreen spectra to derive a stellar velocity field in the center of NGC 5055 by cross-correlating one-element galactic spectra with the spectra of three $K$-giant stars with known line-of-sight velocities. In the red spectral range we measured baricentric positions of the emission line [N II $] \lambda 6583$, which is the strongest in the center of NGC 5055, and also of the $\mathrm{H} \alpha$ emission line, to derive the velocity field of the ionized gas. We estimated the best accuracy of our velocity measurements as $10 \mathrm{~km} \mathrm{~s}^{-1}$ from the night-sky line [O I] $\lambda 6300$ analysis.

In addition to the $2 \mathrm{D}$ spectral data, we used some archive photometric data. NGC 5055 was observed by HST, in particular with NICMOS-CAM3 (ID 7919, PI W. Sparks). The galaxy was exposed on June 4, 1998, during $192 \mathrm{~s}$ through a $F 160 \mathrm{~W}$ (H-continuum) filter and during $512 \mathrm{~s}$ through a $F 187 N(\mathrm{~Pa} \alpha)$ filter. The results of these observations are reported in detail by Boker et al. (1999); we use them to estimate morphological parameters of isophotes. To extend our photometric analysis to the outer parts of the global galactic disk, we also took recourse to ground-based photometry; we used the public data of Frei et al. (1996), obtained at the Palomar $1.5 \mathrm{~m}$ telescope through Thuan \& Gunn's gri-filters: though of medium resolution (seeing of $22^{\prime \prime} 1-22^{\prime \prime} 7$ ), these data are deep and well-calibrated.

\section{Stellar population and ionized gas in the center of NGC 5055}

To study stellar population properties in the center of NGC 5055, we use the Lick indices $\mathrm{H} \beta, \mathrm{Mgb}$, and $\langle\mathrm{Fe}\rangle \equiv$ $(\mathrm{Fe} 5270+\mathrm{Fe} 5335) / 2$. Models of simple stellar populations, e.g. by Worthey (1994), make it possible to determine simultaneouly a luminosity-weighted mean age and a mean metallicity for the stellar populations by comparing $\mathrm{H} \beta$ with $\mathrm{Mgb}$ or $\mathrm{H} \beta$ with $\langle\mathrm{Fe}\rangle$, because the Balmer absorption lines are more sensitive to the age and the metal-line indices are more sensitive to the metallicity.

Figure 1 presents 2D distributions of the Lick indices mentioned above in the center of the galaxy. For a chemically distinct nucleus, both metal-line indices (or sometimes only $\mathrm{Mgb}$ ), have to peak in the photometric center of a galaxy. Indeed, both $\langle\mathrm{Fe}\rangle$ and Mgb vary over the field of view showing maximum values near the center. But whereas the $\langle\mathrm{Fe}\rangle$ peak coincides with the photometric center of the galaxy, the Mgb-enhanced area is shifted to the south-west from the nucleus; it is well resolved, with its own center $\sim 22^{\prime \prime} .5$ from the photometric center of the galaxy. The whole Mgb-enhanced structure looks like one half of a nuclear bar or like one half of a highly inclined circumnuclear disk. Its contrast over the surrounding bulge region, $\Delta \mathrm{Mgb}$ of $0.6-1.0 \AA$, exceeds $3 \sigma$. The $\langle\mathrm{Fe}\rangle$-enhanced area is more symmetric around the center though a weak "plume" toward the Mgb maximum can be noted. Earlier we found different $\mathrm{Mg}$ and $\mathrm{Fe}$ distributions in the centers of galaxies possessing chemically distinct nuclei, but they were different in opposite sense - usually, the Mgb peak was unresolved and coincided with the optical nucleus, and the iron-enhanced areas were more extended and were treated by us as "Fe-rich circumnuclear disks" (see e.g. NGC 1023, Sil'chenko 1999a; NGC 7331, Sil'chenko 1999b; or NGC 4594, Emsellem et al. 1996). The distribution of $\mathrm{H} \beta$, though contaminated by emission at $R>3^{\prime \prime}$, can be used for stellar population analysis in the center; it matches qualitatively the distribution of $\langle\mathrm{Fe}\rangle$ : the enhanced $\mathrm{H} \beta$ absorption peaks in the photometric center of the galaxy. This coincidence is explicable: to obtain a higher iron abundance in stars, the star formation burst has to be long enough to allow the supply of a large amount of iron from SNeIa, and such a long star formation burst would decrease the mean age of the integrated stellar population, thus increasing the $\mathrm{H} \beta$ index. But to determine correctly both age and metallicity, we must use "H $\beta$ vs. metal index" diagrams.

Firstly we must assure ourselves about the magnesiumto-iron ratio in the central stellar population of NGC 5055. If this ratio is not solar, and we apply models with the solar $\mathrm{Mg} / \mathrm{Fe}$ to these data, various metal-line indices, when confronted with $\mathrm{H} \beta$, would give different age estimates. Figure 2 (top) presents a " $\langle\mathrm{Fe}\rangle$ vs. Mgb"diagram to provide the necessary analysis. As was shown by Worthey et al. (1992), in the "iron index vs. magnesium index"-diagrams the models of stellar populations with solar $\mathrm{Mg} / \mathrm{Fe}$ ratio are concentrated within a narrow locus independent of their ages or initial stellar mass function. Any deviation from this locus signifies a nonsolar magnesium-to-iron ratio. Worthey et al. (1992) found that the majority of elliptical galaxies lie to the right of the model locus, being mostly magnesium overabundant. Theoreticians now explain this feature by a main star 

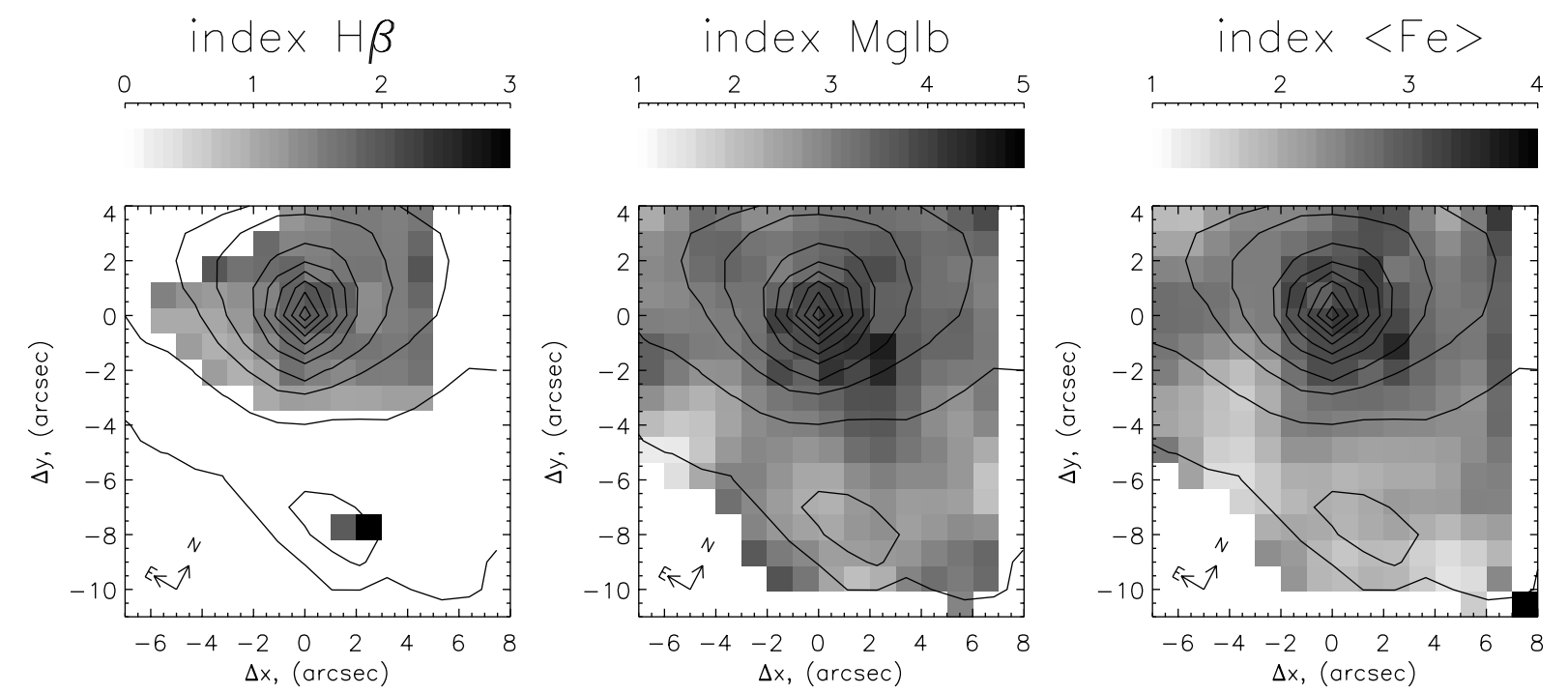

Fig. 1. Lick indices maps (gray-scaled) for the central part of NGC 5055 overlaid by the green continuum isophotes; $<$ Fe $>\equiv$ $(\mathrm{Fe} 5270+\mathrm{Fe} 5335) / 2$. The maps are spatially smoothed by a $2 \mathrm{D}$ Gaussian with $\sigma=0^{\prime \prime} .75$ except the central $5^{\prime \prime} \times 5^{\prime \prime}$.

formation epoch with duration less than 1 Gyr which finished before the bulk of SNeIa exploded. In disk galaxies, the statistics of the magnesium-to-iron ratio is more heterogeneous due perhaps to a larger variety in their circumnuclear evolution. In NGC 5055 (Fig. 2, top) the first impression is that the magnesium-to-iron ratio is close to the solar one and does not change substantially along the radius. But when we look at the next diagram, " $\mathrm{H} \beta$ vs. $\langle\mathrm{Fe}\rangle$ ", or at the diagram of "H $\beta$ vs. [MgFe]" (Fig. 2 middle and bottom), we discover that because of the high $\mathrm{H} \beta$ index in the photometric center of the galaxy the mean age of the nuclear stellar population cannot be larger than 3-4 Gyr. This means that the position of the galactic nucleus, and also of the nearest circumnuclear outskirts, in the diagram " $\langle\mathrm{Fe}\rangle$ vs. Mgb" suggests rather a mild magnesium overabundance if compared to the 5-Gyr model sequence. Therefore, to quantify carefully the population parameters we must also involve the models of Tantalo et al. (1998) calculated for $[\mathrm{Mg} / \mathrm{Fe}]=+0.3$. The middle and bottom parts of Fig. 2 present age-diagnostic diagrams for $[\mathrm{Mg} / \mathrm{Fe}]=+0.3$ (the models of Tantalo et al. 1998) and for $[\mathrm{Mg} / \mathrm{Fe}]=0$ (the models of Worthey 1994); the right value of the age lies between the estimates made from these two diagrams. To use the absorption-line in$\operatorname{dex} \mathrm{H} \beta$ for the stellar population diagnostics, we must correct it for the emission contamination which is not negligible at $R>3^{\prime \prime}$. We have done it in the following way: we have summed the red spectra in the same concentric rings as the green ones, have calculated the equivalent widths of the $\mathrm{H} \alpha$ emission line, $E W_{\mathrm{H} \alpha}(R)$, and then have estimated the correction for the $\mathrm{H} \beta$ emission as $E W_{\mathrm{H} \beta, \mathrm{em}}=0.25 E W_{\mathrm{H} \alpha, \mathrm{em}}$ (Stasinska \& Sodre 2001). The values of $\mathrm{H} \beta$ indices in Fig. 2 have all been corrected for the emission. By inspecting the Fig. 2, middle and bottom, we conclude that the mean ages of the stellar populations in the nucleus and in the magnesium-enhanced region to the SW of the nucleus are roughly the same and are certainly less than 5 Gyr; we estimate them as 3-4 Gyr. The mean metallicity of the nucleus is higher than the solar, $[\mathrm{Fe} / \mathrm{H}]_{\mathrm{nuc}} \approx+0.2-+0.3$, and the mean metallicity of the SW magnesium-rich "island" is higher by $0.2-0.3$ dex than that of the nucleus. Farther from the center, the mean age of the stellar population rises sharply and the $\mathrm{Mg} / \mathrm{Fe}$ ratio approaches the solar one. Basing ourselves mainly on the models of Worthey (1994) (Fig. 2, bottom) we estimate the mean age of the stellar population in the bulge of NGC 5055, at $R=2^{\prime \prime}-5^{\prime \prime}$, as 8-10 Gyr.

To illustrate how severely the $\mathrm{H} \beta$ index is contaminated by Balmer emission in the central region of NGC 5055 and to give an impression of the ionized gas distribution and excitation, we present surface brightness distributions of the red emission line intensities in Fig. 3. The $\mathrm{H} \alpha$ emission is negligible in the nucleus (see also Pogge's, 1989, statement that NGC 5055 lacks emission lines in the nucleus), but there are several bright spots, including one on the major axis at $\sim 5^{\prime \prime}$ to the east of the nucleus and one at $9^{\prime \prime}$ to the south-west of the nucleus. The latter H II region was earlier detected and noted in their Conclusions by Pismis et al. (1995). Therefore, though NGC 5055 is known as a LINER, it also possesses "hot spots" - sites of intense star formation? - in its circumnuclear area. The intensity distribution of $[\mathrm{N} \mathrm{II}] \lambda 6583$ is peaked in the nucleus as expected for the LINER. However, this distribution is noticeably asymmetric around the center, the eastern part being brighter. If we remember the asymmetry of the stellar kinematics along the major axis of NGC 5055 reported by Fillmore et al. (1986) and its interpretation as bulge obscuration by dust to the west of the nucleus, we have to conclude that the $[\mathrm{NII}]$ emission is probably related mostly to the bulge. Besides, the doubt of Fillmore et al. (1986) that the weaker absorption lines to the east of the nucleus contradict the dynamical arguments for the bulge 

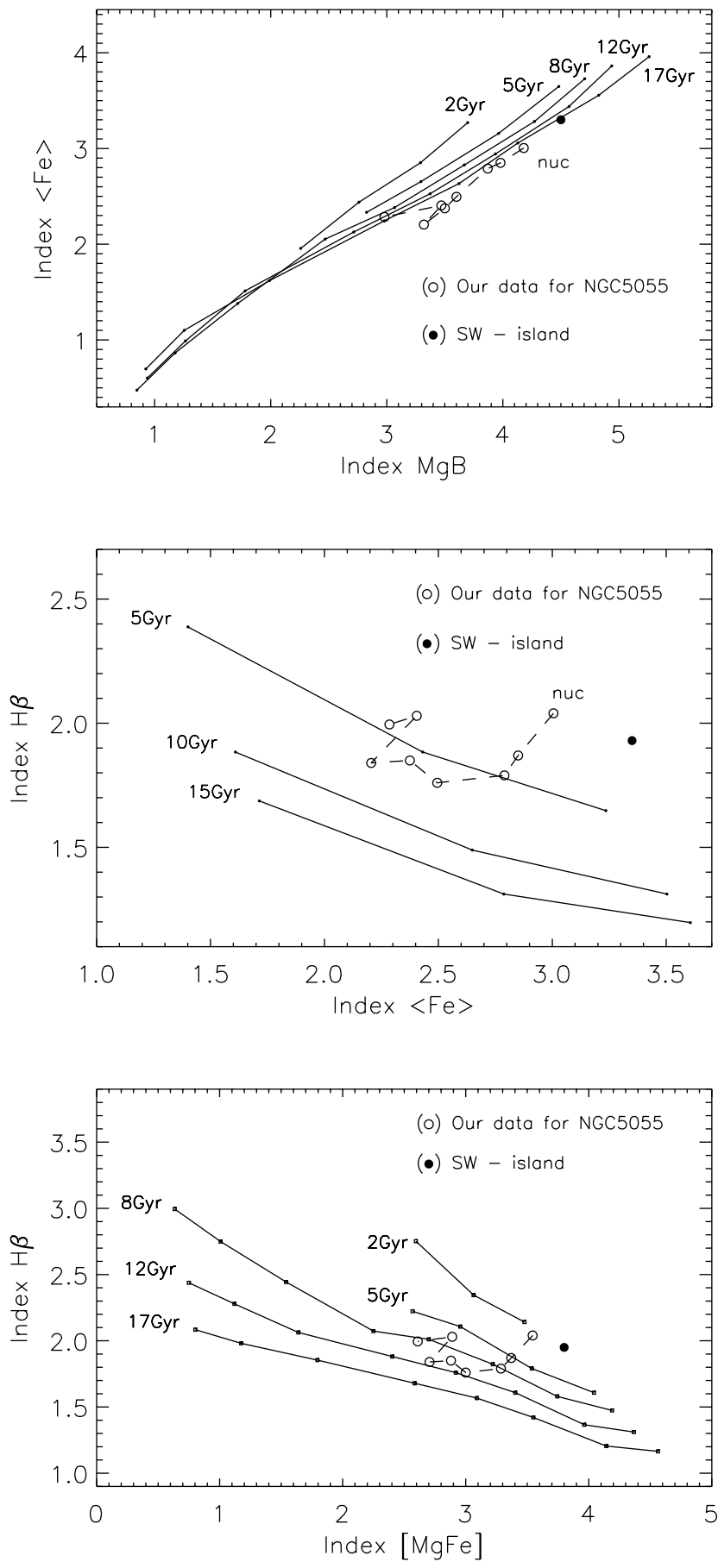

Fig. 2. "Index-index" diagnostic diagrams for the azimuthally averaged Lick indices in the center of NGC 5055 taken along the radius in steps of $1^{\prime \prime}$ (open circles); (top) < Fe $>$ vs. Mgb diagram, with the models of Worthey (1994) for $[\mathrm{Mg} / \mathrm{Fe}]=0$, (middle) $\mathrm{H} \beta$ vs. $<\mathrm{Fe}>$, with the models of Tantalo et al. (1998) for $[\mathrm{Mg} / \mathrm{Fe}]=+0.3$, and (bottom) $\mathrm{H} \beta$ vs. $[\mathrm{MgFe}] \equiv$ $(\mathrm{Mgb}<\mathrm{Fe}>)^{1 / 2}$, with the models of Worthey (1994) for $[\mathrm{Mg} / \mathrm{Fe}]=0$. In addition to the azimuthally averaged data we have also plotted the Mg-rich compact region to the south-west of the nucleus ("SW-island") by a black dot. Small signs connected by thin lines present stellar population models of equal ages; the metallicities for the Worthey's models are +0.50 , $+0.25,0.00,-0.22,-0.50,-1.00,-1.50,-2.00$, if one takes the signs from the right to the left, and for the models of Tantalo et al. they are $+0.4,0.0$, and -0.7 . visibility here now can be put away because of the detection of the chemically distinct nucleus in the form of an asymmetric circumnuclear disk-like structure.

\section{Kinematics of the stars and ionized gas in NGC 5055}

The 2D spectroscopy provides us with full line-of-sight velocity fields for both stars and ionized gas that are much more informative than long-slit cross-sections. Figure 4 presents isovelocities of the ionized gas mapped by measuring independently the $\mathrm{H} \alpha$ and [N II] $\lambda 6583$ emission lines. The velocity field looks rather regular and reflects mostly plane gas rotation; a faster rotation implied by the $\mathrm{H} \alpha$ measurements may be an artifact of the strong stellar $\mathrm{H} \alpha$ absorption line and slower stellar rotation with respect to the ionized gas. Figure 5 gives the distributions of the stellar line-of-sight velocities and stellar velocity dispersion. Indeed, stars rotate slightly slower than the ionized gas. The disturbance of the stellar velocity field to the south-west of the center seems to be stronger than the similar isovelocity twisting in Fig. 4, but in general we may conclude that the velocity anomaly at $R \approx 3^{\prime \prime}$ to the west and south-west of the nucleus which was reported by Pismis et al. (1995) for the ionized gas, is found to be present in the velocity distributions of both the stars and the ionized gas. The stellar velocity dispersion distribution (Fig. 5, right) also looks asymmetric: there is a local minimum of the velocity dispersion in the nucleus, and its maximum area has an arc-like shape and is prominent to the west of the photometric center. We find this rather puzzling.

Whereas long-slit kinematical techniques can in general provide information about real motions of stars and gas only on a priori assumptions, usually on the assumption of circular (axisymmetric) rotation, the $2 \mathrm{D}$ velocity fields give a possibility to diagnose the character of these motions, in particular, to verify the validity of the circular rotation paradigm. If we have an axisymmetric mass distribution, and rotation on circular orbits, the direction of maximum central line-of-sight velocity gradient (we shall call it "kinematical major axis") should coincide with the line of nodes as well as the photometric major axis; whereas in the case of a triaxial potential the isovelocities align with the principal axis of the ellipsoid, and generally the dynamical and photometrical major axes diverge, turning in opposite senses with respect to the line of nodes (e.g. Monnet et al. 1992; Moiseev \& Mustsevoy 2000). In the simple case of cylindric (disk-like) rotation we have a convenient analytic expression for the azimuthal dependence of the central line-of-sight velocity gradient within the area of solid-body rotation:

$\mathrm{d} v_{\mathrm{r}} / \mathrm{d} r=\omega \sin i \cos \left(\mathrm{PA}-\mathrm{PA}_{0}\right)$

where $\omega$ is the deprojected central rotation angular velocity, $i$ is the inclination of the rotation plane, and $\mathrm{PA}_{0}$ 

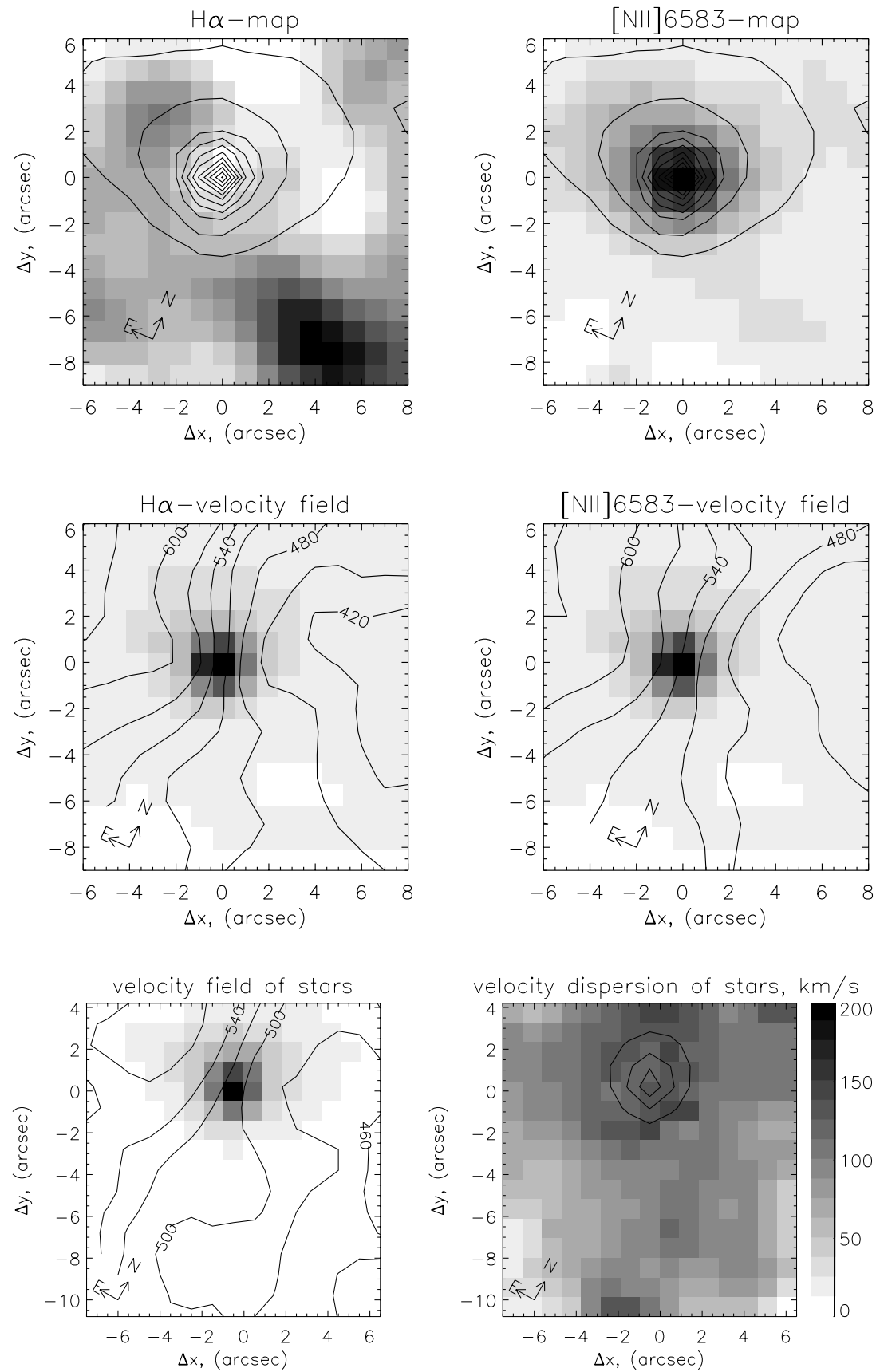
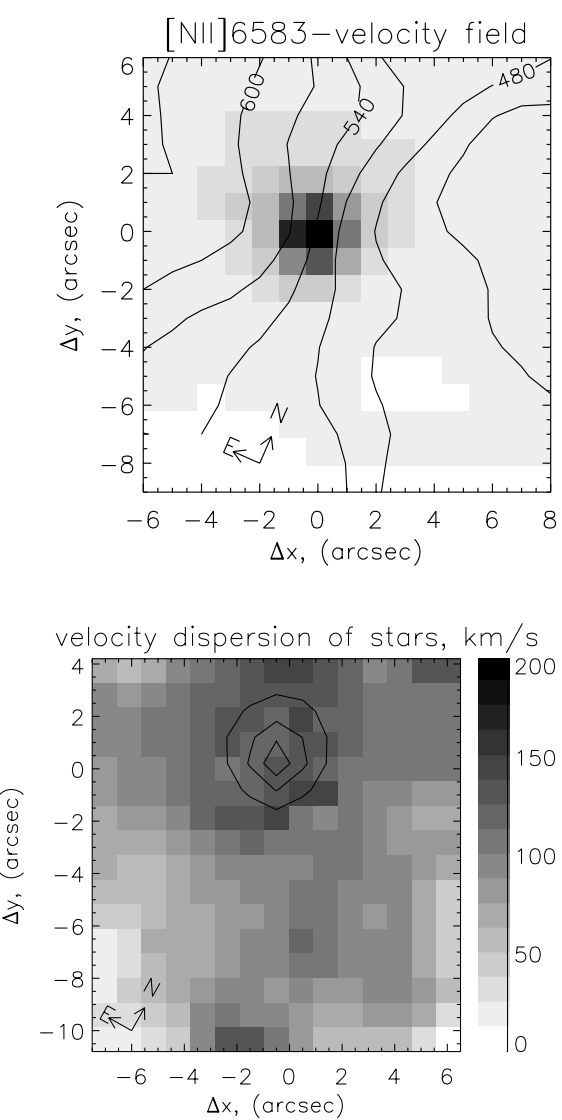

Fig. 3. Emission-line intensity maps (grayscaled) for the central part of NGC 5055 overlaid by the red continuum isophotes.

Fig. 4. Ionized-gas line-of-sight velocity fields in the central part of NGC 5055 (isolines) as measured by the $\mathrm{H} \alpha$ and $\mathrm{N}[\mathrm{II}] \lambda 6583$ emissionline baricenter positions. The gray-scaled background presents the red continuum intensity.

Fig. 5. The line-of-sight velocity field of the stellar component (left, isolines) and the stellar velocity dispersion map (right, gray-scaled) in the central part of NGC 5055. The continuum intensity is shown as gray-scaled on the left plot and as isolines on the right plot. is the orientation of the line of nodes, coinciding in the case of the axisymmetric ellipsoid (or a thin disk) with the photometric major axis. So by fitting azimuthal variations of the central line-of-sight velocity gradients with a cosine curve, we can determine the orientation of the kinematical major axis by its phase and the central rotation angular velocity by its amplitude.

Figure 6 presents the azimuthal variations of the lineof-sight velocity gradients in a radius ranging from 1 ." 5 to 4 .. 0 for the stars and for the ionized gas, which are traced by $\mathrm{H} \alpha$ and $[\mathrm{NII}] \lambda 6583$ emission lines; Table 3 contains the parameter values obtained by fitting these measurements by a cosine function in somewhat narrower radial bins. First of all, one can see from Table 3 that due to the good seeing quality we detect a certain decrease of the gas-rotation angular velocity over a range of $1^{\prime \prime}-5^{\prime \prime}$ in radius. We do therefore not confirm the result of Pismis et al. (1995) concerning solid-body gas rotation of NGC 5055 up to the radius of $3^{\prime \prime}$. As the solid-body rotation area is traced by us to at most $R=1^{\prime \prime} .5$ (and actually it is even smaller), the formula for the azimuthal velocity gradient variations given above ceases to be precise, but still remains approximately valid, because due to small ellipticity of isophotes in the center of NGC 5055 (Fig. 7) the projected radius is close to the true one. Indeed, the measurements in Fig. 6 are well fitted by a cosinusoid. We just note one feature of the azimuthal dependencies of Fig. 6 : in the position angle range of $230^{\circ}-310^{\circ}$ all the plots demonstrate weaker or stronger deviations from the cosinusoids, in other words, from circular rotation in the sense that 
Table 3. Parameters of the azimuthal velocity-gradient variations fitting.

\begin{tabular}{|c|c|c|c|}
\hline Component & Radius range of fitting & $\overline{\overline{\mathrm{PA}_{0}}}$ & $\overline{c \omega \sin i, \mathrm{~km} \mathrm{~s}^{-1} \operatorname{arcsec}^{-1}}$ \\
\hline Ionized gas & $0^{\prime \prime} 9-1^{\prime \prime} .3$ & $100^{\circ} \pm 1^{\circ}$ & $65 \pm 9$ \\
\hline Stars & $1^{\prime \prime} 4-2^{\prime \prime} 3$ & $97^{\circ} \pm \ldots$ & $22 \pm 6$ \\
\hline Ionized gas & $1^{\prime \prime} 9-2^{\prime \prime} .7$ & $104^{\circ} \pm 3^{\circ}$ & $31 \pm 9$ \\
\hline Stars & $2^{\prime \prime} 4-3^{\prime \prime} .7$ & $95^{\circ} \pm \ldots$ & $15 \pm 4$ \\
\hline Ionized gas & $2^{\prime \prime} 8-4^{\prime \prime} .0$ & $103.5^{\circ} \pm 0.5^{\circ}$ & $25 \pm 8$ \\
\hline Ionized gas & $3 .{ }^{\prime \prime} 8-5^{\prime \prime} .3$ & $100^{\circ} \pm 7^{\circ}$ & $21 \pm 6$ \\
\hline
\end{tabular}

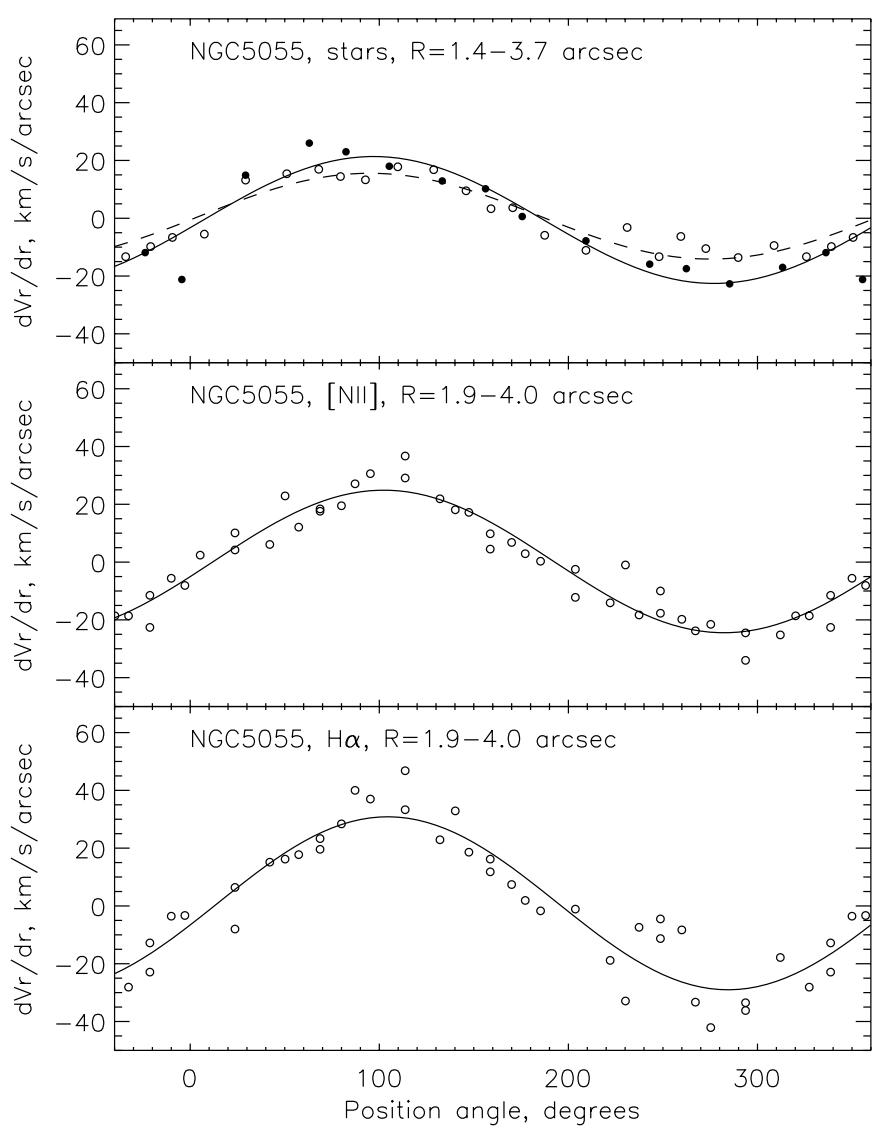

Fig. 6. The azimuthal dependencies of the line-of-sight velocity gradients for the stars (top) and for the ionized gas (middle and bottom) in the center of NGC 5055 fitted by cosine curves with a least-square method. Note the deviations from the cosine law (or from circular flat rotation) at the line of nodes. For the stars, black dots show the measurements in the $R=11^{\prime \prime} 4-2^{\prime \prime} 3$ range, and open circles represent the measurements in the $R=$ $2^{\prime \prime} 4-3^{\prime \prime} 7$ range; a dashed-line cosine curve fitting formally the latter data demonstrates a lower rotation-velocity amplitude but is it a real effect? The maximum discrepancy between the two data sets is observed at $\mathrm{PA}=230^{\circ}-310^{\circ}$, so it is perhaps the same deviation from a pure circular rotation that is seen at the bottom plot.

there is a deficiency of the velocity at $\mathrm{PA}<280^{\circ}$ and an excess at $\mathrm{PA}>280^{\circ}$. We think that just this effect has been observed by Pismis et al. (1995) in their long-slit cross-sections. We now have a full two-dimensional picture, though, and it becomes clear that this cannot be a radial gas outflow; it may be either counterrotating gas

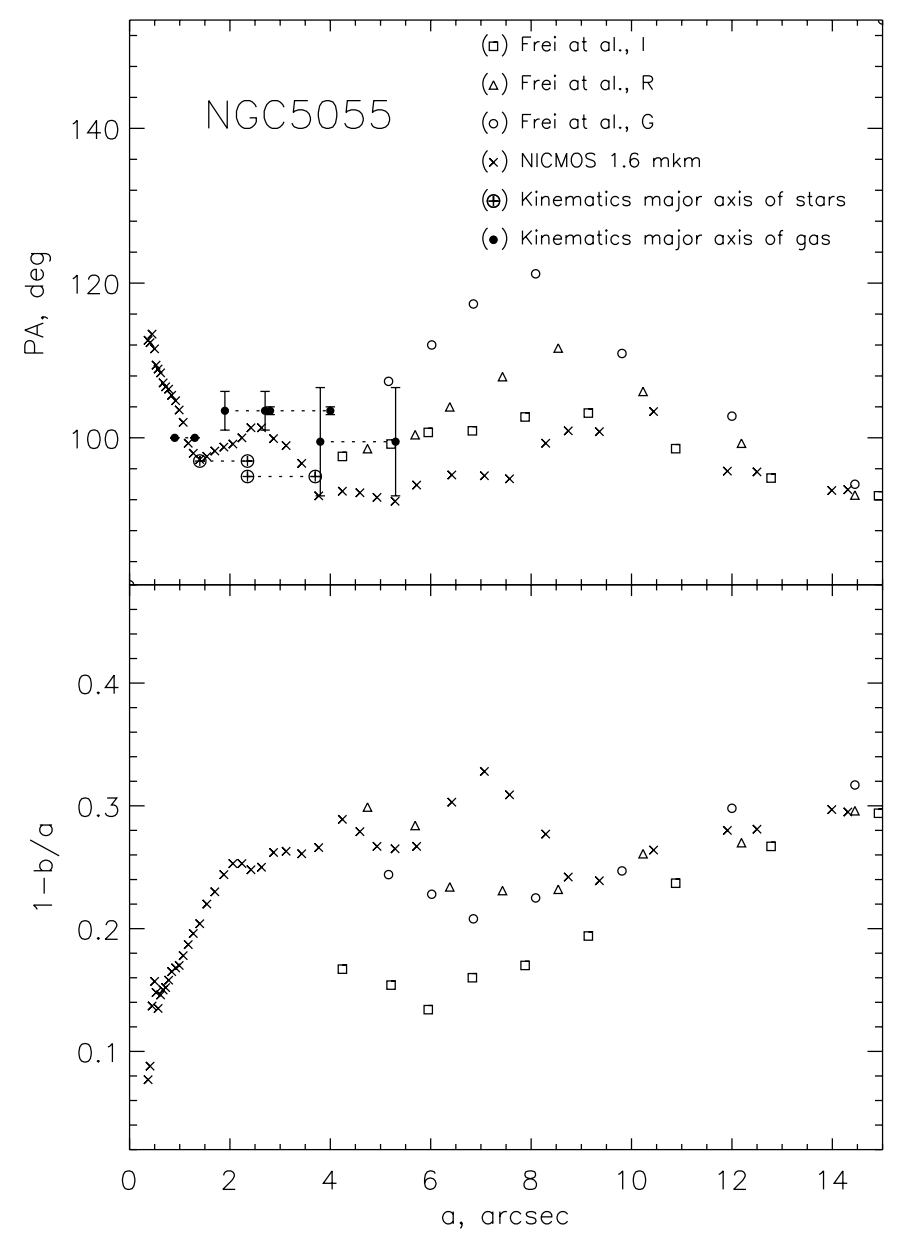

Fig. 7. Isophote characteristics together with the orientations of the kinematical major axes for the stars and ionized gas in the center of NGC 5055. The line of nodes determined from the outermost isophote orientation is $\mathrm{PA}=105^{\circ}$ and the galaxy plane inclination of $55^{\circ}$ corresponds to $(1-b / a)_{0}=0.43$.

streaming around a thick minibar roughly aligned with the line of nodes, or, more probably, a gas polar arc (ring?) shifted from the center and perhaps wrapped around the same minibar.

It remains unclear if there is really a minibar in the center of NGC 5055. On the one hand, the morphology of the Mgb surface distribution, the presence of the H II spot at the major axis, and the kinematical disturbances described above constitute evidence for it. On the other hand, we find that the orientations of the kinematical and photometric major axes in the center of NGC 5055 (Fig. 7) 
are coincident. Both may deviate slightly from the line of nodes of the outer disk, $\mathrm{PA}_{0}=105^{\circ}$, but they do it together. Only one possibility for a bar remains under such circumstances: if it is fully aligned with the line of nodes of the circumnuclear rotation plane. But in such a configuration the velocity profile along the major axis must have a plateau in the center and it does not have one. The problem remains to be solved.

\section{Discussion}

The kinematical and stellar population parameter distributions in the center of NGC 5055 have been shown to be so complex that they cannot yet be interpreted unambiguously. Among a dozen spiral galaxies with chemically distinct nuclei studied by us with the MPFS, we have not seen any analogous cases. In the absence of the observational counterparts, a good choice would be to sketch an environment of this complex central structure and to try to understand what should proceed inevitably in such environment from the dynamical point of view. But as we mentioned in the Introduction, the whole structure of NGC 5055 is highly ambiguous. Our own attempts to clarify it have not been very productive. The brightness profile in the range of radius of $2^{\prime \prime}-12^{\prime \prime}$ derived from the NICMOS/HST data is well-fitted by a de Vaucouleurs' formula indicating a classical bulge. But in the same range of radius the ionized gas spirals are seen - mainly to the west of the nucleus (Boker et al. 1999); therefore for some reason the prominent bulge is not able to stabilize the inner disk. Could the bulge be non-axisymmetric? Its major axis is aligned in $\mathrm{PA} \approx 95^{\circ}$ so being turned by $10^{\circ}$ with respect to the line of nodes, and the ellipticity is constant but low (Fig. 7). But the kinematical major axes of the stars and ionized gas trace the photometric major axis with a high precision instead of being turned by the same $10^{\circ}$ in opposite sense (Monnet et al. 1992); so they rotate axisymmetrically. Is it due to a possible strong mass concentration in the very center? All these questions remain still unclarified. Now we can only imagine some possible configurations in the center of NGC 5055 and give a general qualitative description.

1. A lopsided circumnuclear stellar disk.

A wave perturbation with an $m=1$ has now become a popular field of consideration. Theoreticians predicted one-sided bars (e.g. Colin \& Athanassoula 1989) and eccentric nuclei (e.g. Miller \& Smith 1992) some time ago, one-armed spirals were also favoured by some dynamical models. Recently a growing amount of observational data has begun to confirm the reality of such structures, in particular in the centers of normal spiral galaxies. The most famous lopsided circumnuclear stellar disk belongs to M 31: its two brightness centers, one with high stellar velocity dispersion and the other dynamically cold, have been explained by Tremaine (1995) as an eccentric Keplerian disk around a supermassive black hole, and Bacon et al. (2001) have argued that this disk must precess with an angular velocity of some $3 \mathrm{~km} \mathrm{~s}^{-1} \mathrm{pc}^{-1}$ so suffering an $m=1$ mode. In NGC 5055 there are too many asymmetries along the major axis: the brightness asymmetry, including [N II] and $\mathrm{H} \alpha$ emission lines, the rotation and stellar velocity dispersion asymmetries, and finally, the Mgb index distribution asymmetry found by us in this work. Whereas a sole brightness asymmetry can always be explained by the dust projection effect (though it is usually more pronounced along the minor axis), the whole complex of asymmetries seen in the center of NGC 5055 proves its intrinsic physical reality; in particular, the dust cannot affect narrow-band spectral features, such as the Mgb index, and cannot create artificial Mgb enhancement to the south-west from the center. We can imagine a chemically distinct, rather young circumnuclear stellar disk traced by the high Mgb index; it can precess slowly so that stars born, say, a few Gyr ago at the eastern circumnuclear H II region are now to the west of the dynamical center. We know that there is a mass concentration in the nucleus of NGC 5055 because of the rotation velocity peak near the center; it may be a compact young stellar cluster (Maoz et al. 1995) or a supermassive black hole, so the whole situation may be similar to that in M 31, though at a larger scale.

2. Another possibility which always helps to resolve a complex situation is a minor merger. We could identify the magnesium-enhanced site with the core of the merged galaxy and call it "a secondary nucleus". But in this particular case the hypothesis of minor merger meets a lot of problems. The fact of the exact coincidence of the mean ages of stellar populations in the primary and secondary nuclei looks somewhat strange. Did the nuclei host synchronous star formation bursts prior to merging? Moreover, the "secondary" nucleus has a metallicity twice as high as that of the "primary" nucleus; more metal-rich nuclei belong usually to more luminous and massive galaxies; if so, the merger could not be a "minor" one. And finally, as we see a clear azimuthal asymmetry of the merger remnants distribution, the event should be very young, not older than one orbital period, $\sim 10^{7} \mathrm{yr}$, and then we should see some other signatures of merging: tidal tails, global disk heating and disappearance of spiral structure, etc. We see none.

So the first hypothesis seems to be preferable.

Acknowledgements. We thank the post-graduate student of SAO RAS A. V. Moiseev for supporting the observations at the $6 \mathrm{~m}$ telescope. The $6 \mathrm{~m}$ telescope is operated under the financial support of Science Ministry of Russia (registration number 01-43). During the data analysis we have used the Lyon-Meudon Extragalactic Database (LEDA) supplied by the LEDA team at the CRAL-Observatoire de Lyon (France) and the NASA/IPAC Extragalactic Database (NED) which is operated by the Jet Propulsion Laboratory, California Institute of Technology, under contract with the National Aeronautics and Space Administration. The research is partly based on observations made with the NASA/ESA Hubble Space Telescope, 
obtained from the data archive at the Space Telescope Science Institute, which is operated by the Association of Universities for Research in Astronomy, Inc., under NASA contract NAS 5-26555. The work was supported by the grant 1.2.4.1 of the Russian State Scientific-Technical Program "Astronomy. Basic Space Researches" (the "Astronomy" section).

\section{References}

Acaretta, J. R., Manteiga, M., Pismis, P., Mampaso, A., \& Cruz-Gonzalez, G. 1996, AJ, 112, 1894

Afanasiev, V. L., Vlasyuk, V. V., Dodonov, S. N., \& Sil'chenko, O. K. 1990, Preprint SAO N54, Nizhnij Arkhyz: Special Astrophys. Obs.

Afanasiev, V. L., Dodonov, S. N., Drabek, S. V., \& Vlasyuk, V. V. 1996, MPFS Manual. Nizhnij Arkhyz: SAO Publ.

Bacon, R., Emsellem, E., Combes, F., et al. 2001, A\&A, 371, 409

Baggett, W. E., Baggett, S. M., \& Anderson, K. S. J. 1998, AJ, 116,1626

Boker, T., Calzetti, D., Sparks, W., et al. 1999, ApJS, 124, 95

Bosma, A. 1981, AJ, 86, 1791

Burbidge, E. M., Burbidge, G. R., \& Prendergast, K. H. 1960, ApJ, 131, 282

Cardiel, N., Gorgas, J., Cenarro, J., \& Gonzalez, J. J. 1998, A\&AS, 127, 597

Colin, J., \& Athanassoula, E. 1989, A\&A, 214, 99

Elmegreen, D. M. 1981, ApJS, 47, 229

Elmegreen, D. M., \& Elmegreen, B. G. 1987, ApJ, 314, 3

Emsellem, E., Bacon, R., Monnet, G., \& Poulain, P. 1996, A\&A, 312, 777
Fillmore, J. A., Boroson, T. A., \& Dressler, A. 1986, ApJ, 302, 208

Fish, R. A. 1961, ApJ, 134, 880

Frei, Z., Guhathakurta, P., Gunn, J. E., \& Tyson, J. A. 1996, AJ, 111, 174

Heraudeau, P., \& Simien, F. 1996, A\&AS, 118, 111

Heraudeau, P., \& Simien, F. 1998, A\&AS, 133, 317

Kent, S. M. 1987, AJ, 93, 816

Maoz, D., Filippenko, A. V., Ho, L. C., et al. 1995, ApJ, 440, 91

Miller, R. H., \& Smith, B. F. 1992, ApJ, 393, 508

Moiseev, A. V., \& Mustsevoy, V. V. 2000, Pis'ma v AZh, 26, 657

Monnet, G., Bacon, R., \& Emsellem, E. 1992, A\&A, 253, 366

Pismis, P., Mampaso, A., Manteiga, M., Recillas, E., \& Cruz-Gonzalez, G. 1995, AJ, 109, 140

Pogge, R. W. 1989, ApJS, 71, 433

Sil'chenko, O. K. 1994, AZh, 71, 706

Sil'chenko, O. K. 1999a, AJ, 117, 2725

Sil'chenko, O. K. 1999b, AJ, 118, 186

Sil'chenko, O. K., \& Afanasiev, V. L. 2000, A\&A, 364, 479

Stasinska, G., \& Sodre, Jr. I. 2001, A\&A, 374, 919

Tantalo, R., Chiosi, C., \& Bressan, A. 1998, A\&A, 333, 419

Thornley, M. D. 1996, ApJ, 469, L45

Thornley, M. D., \& Mundy, L. G. 1997, ApJ, 484, 202

Tremaine, S. 1995, AJ, 110, 628

Vlasyuk, V. V. 1993, Astrofiz. issled. (Izv. SAO RAS) 36, 107

Worthey, G. 1994, ApJS, 95, 107

Worthey, G., Faber, S. M., \& Gonzalez, J. J. 1992, ApJ, 398, 69

Worthey, G., Faber, S. M., Gonzalez, J. J., \& Burstein, D. 1994, ApJS, 94, 687 